\title{
Perfil sócio demográfico e áreas de desempenho ocupacional afetadas em pacientes pós-AVE atendidos por um serviço de terapia ocupacional
}

\author{
Socio-demographic profile and areas of \\ occupational performance in stroke patients \\ attended by occupational therapy service
}

\author{
Kátia Vanessa Pinto de Meneses ${ }^{1}$, Jeanine Schuabb Duarte ${ }^{2}$, Vanessa \\ de Oliveira Alencar ${ }^{3}$, Ana Carolina dos Santos Pereira ${ }^{4}$
}

\begin{abstract}
MENESES, K. V. P.; DUARTE, J. S.; ALENCAR, V. O.; PEREIRA, A. C. S. .Perfil sócio demográfico e áreas de desempenho ocupacional afetadas em pacientes pós-ave atendidos por um serviço de terapia ocupacional. Rev. Ter. Ocup. Univ. São Paulo, v. 23, n. 2, p. 107-112, maio/ ago. 2012.
\end{abstract}

RESUMO: Introdução: As seqüelas do acidente vascular encefálico (AVE) resultam em dificuldade ou incapacidade para realizar tarefas funcionais e do dia a dia, dificultando ou impossibilitando o retorno ao trabalho, a participação na família e na comunidade. Intervenções de terapia ocupacional ajudam a melhorar as habilidades nas atividades de vida diária, favorecendo maior independência, autonomia, participação social e qualidade de vida. Objetivo: Identificar o perfil sócio demográfico de pacientes pós AVE atendidos em um serviço de terapia ocupacional e identificar as áreas de desempenho ocupacional mais afetadas. Metodologia: Estudo transversal, com pacientes pósAVE. Foi utilizada uma entrevista para coleta de informações sócio demográficas e a Medida Canadense de Desempenho Ocupacional (COPM). Resultados: 19 pacientes, sendo $9(47,3 \%)$ do sexo feminino e $10(52,6 \%)$ do sexo masculino, idade entre 36 e 74 anos $(20-39=21 \%$; 40-60 = $57,89 \% ;>60=21 \%) .13(68,4 \%)$ eram casados ou possuíam união estável. A maioria apresentou ensino fundamental incompleto. Os indivíduos relataram 40 áreas problemas de desempenho ocupacional, sendo as mais citadas alimentação e marcha (autocuidado), cozinhar (produtividade) e escrever (atividades de lazer). Considerações finais: Os resultados apontam para a importância de abordagens reabilitadoras que foquem nestas áreas de desempenho mais afetadas pelo AVE, de forma a possibilitar maior independência, autonomia e participação social.

DESCRITORES: Acidente vascular encefálico/reabilitação; Acidente vascular encefálico/terapia; Perfil de impacto da doença; Terapia ocupacional.

${ }^{1}$ Docente do Curso de Terapia Ocupacional da Universidade de Brasília.

${ }^{2}$ Acadêmica do curso de Terapia Ocupacional e Bolsista de Iniciação Científica pela Universidade de Brasília.

${ }^{3}$ Acadêmica do curso de Terapia Ocupacional e Bolsista de Iniciação Científica pela Universidade de Brasília.

${ }^{4}$ Acadêmica do curso de Terapia Ocupacional da Universidade de Brasília.

Endereço para correspondência: Kátia Vanessa Pinto de Meneses. Av. Flamboyant, lote 22, bloco C, apto 1101, Águas Claras, Brasília, DF, CEP 71917000. E-mail.: katiavpmeneses@gmail.com 


\section{INTRODUÇÃO}

$\mathrm{O}$ acidente vascular encefálico (AVE) representa uma das causas mais comuns de morte em todo o mundo e a principal causa de incapacidade e invalidez (LAVADOS et al., 2007; POLESE et al., 2008, DOBKIN, 2004). Segundo Lavados et al. (2007), dois terços das mortes provocadas pelo AVE ocorrem em países subdesenvolvidos e em desenvolvimento, como em países da América Latina e do Caribe. Estudos recentes, mostram que a incidência de mortes por doenças cardiovasculares, incluindo o AVE, vem diminuindo nos últimos 30 anos em países da América Latina, podendo este evento estar relacionado ao aumento socioeconômico nesses países, que promove a diminuição da exposição aos fatores de riscos clássicos e a melhora na qualidade do tratamento (CURIONI et al., 2009). Estudo realizado no Brasil mostrou declínio da mortalidade por AVE em homens e mulheres, em todas as faixas etárias e em todas as regiões, sendo o maior declínio em áreas mais ricas como a região sul e sudeste do Brasil (ANDRÉ et al., 2006).

As desigualdades sócio econômicas podem ser responsáveis pela alta taxa de mortalidade nas áreas mais pobres do Brasil, uma vez que esses fatores estão diretamente relacionados com a saúde e qualidade de vida, como a falta de educação, a falta de moradia adequada, a qualidade da água utilizada para consumo, a falta de transporte e o difícil acesso nos serviços de saúde. De acordo com Lavados et al. (2007), a significante proporção de AVE`s nas regiões mais pobres se deve à pouca prevenção dos fatores de risco.

Apesar da incidência do AVE aumentar à medida que o ser humano envelhece, este pode ocorrer em diferentes fases da vida (ARES, 2003). Nas décadas passadas, pessoas acima de 65 anos eram mais acometidas pelo AVE, porém ultimamente, tem-se percebido um aumento de adultos jovens nos centros de reabilitação (ARES, 2003). Este fato deve-se a exposição exagerada desses jovens adultos a fatores de risco como histórico familiar, doenças cardíacas, tabagismo, o uso de álcool e outras drogas, o uso de contraceptivos orais, estresses causados pelo cotidiano agitado, entre outros (ARES, 2003).

As seqüelas provocadas pelo AVE trazem conseqüências cognitivas e/ou sensório motoras, que alteram a capacidade funcional, levando à dificuldade ou incapacidade para realizar tarefas do dia a dia, dificultando ou impossibilitando o retorno ao trabalho, alterando a autonomia, independência, a participação na família e na comunidade (CRUZ; DIOGO, 2009). De acordo com Barros (2003), entre os indivíduos que sobrevivem a um AVE, $15 \%$ não apresentam prejuízo de sua capacidade funcional, 37\% mostram discreta alteração e são capazes de realizar atividades de auto cuidado, $16 \%$ apresentam moderada incapacidade, sendo semi-dependentes nas AVDs e $32 \%$ demonstram sequelas graves, diminuindo consideravelmente sua capacidade funcional.

A reabilitação eficaz, dos pacientes com seqüelas de um AVE, inclui uma equipe multidisciplinar da área da saúde com atendimentos que comecem, em situação ideal, 48 horas após a estabilidade do paciente (DOBKIN, 2004). Neste contexto a terapia ocupacional tem como objetivo engajar esses pacientes em atividades que são significativas e importantes para eles, com a finalidade de retorno à independência, à autonomia (capacidade de exercer escolhas e de tomar decisões, por si próprio) e à participação social. As áreas ocupacionais envolvem as atividades básicas da vida diária (de auto-cuidado) e instrumentais (de resolução de problemas complexos e cuidados com o outro), bem como o trabalho, o estudo, o brincar, o lazer e a participação social. Essas áreas podem variar de acordo com a idade da pessoa, o tipo de atividades que realiza, suas preferências, rotina, hábitos, dentre outros. Para o terapeuta ocupacional, interessa o desempenho do ser humano em suas funções e ações do cotidiano (CRUZ; TOYODA, 2009).

Assim, conhecer as áreas de desempenho ocupacional mais acometidas após o AVE é importante para se orientar o uso de técnicas, métodos e intervenções adequadas durante o processo de reabilitação em terapia ocupacional, de forma a promover a melhora da funcionalidade e da qualidade de vida dos pacientes atendidos.

O objetivo deste estudo é traçar o perfil sócio demográfico de indivíduos pós AVE atendidos por um serviço de terapia ocupacional e identificar as áreas de desempenho ocupacional mais afetadas.

\section{MATERIAIS E MÉTODOS}

Tipo de estudo: estudo transversal

População: indivíduos pós-AVE, de ambos os sexos, com idade igual ou superior a 18 anos, sem restrição quanto à classe social, atendidos por um serviço de terapia ocupacional.

Amostra: todos os indivíduos atendidos no Serviço de Terapia Ocupacional da Unidade Mista de Taguatinga, Distrito Federal, no período de agosto de 2010 a julho de 2011.

Local do estudo: O Serviço de Terapia Ocupacional da Unidade Mista de Taguatinga está ligado à Secretaria 
MENESES, K. V. P. et al. Perfil sócio demográfico e áreas. Rev. Ter. Ocup. Univ. São Paulo, v. 23, n. 2, p. 107-112, maio/ago. 2012.

de Estado da Saúde do Distrito Federal e é referência em reabilitação de indivíduos pós-AVE na região.

Critérios de inclusão: indivíduos de ambos os sexos, com idade igual ou superior a 18 anos, sem restrição quanto à classe social, atendidos no Serviço de Reabilitação em Terapia Ocupacional da Unidade Mista de Taguatinga (UMT), Distrito Federal e que assinaram o Termo de Consentimento Livre e Esclarecido (TCLE).

Critérios de exclusão: idade inferior a 18 anos; incapacidade de comunicação ou limitação para expressar suas perspectivas, desde que não fosse acompanhado por um familiar ou intérprete, que compreendesse de perto a situação do cliente; déficit cognitivo que impedisse a compreensão do instrumento utilizado; não concordância em assinar o TCLE.

Coleta de dados: os dados foram coletados por meio de entrevista estruturada contendo informações sócio demográficas e pela Medida Canadense de Desempenho Ocupacional (COPM), para identificar as áreas de desempenho mais acometidas. De acordo com Law et al. (2009), a COPM é utilizada para identificar as ocupações que os clientes querem, precisam ou esperam fazer e avaliar o desempenho desses, bem como sua satisfação no desempenho das ocupações.

Procedimentos: Foi realizado um convite a todos os indivíduos atendidos no serviço de terapia ocupacional da Unidade Mista de Taguatinga, Distrito Federal, no período de agosto de 2010 a julho de 2011, para participarem deste estudo. Aqueles que concordaram em participar e assinaram o TCLE passaram por uma entrevista realizada por duas alunas do curso de terapia ocupacional da Universidade de Brasília (UnB) que foram capacitadas para esta tarefa.

Este estudo foi protocolado sob $n^{\circ} 178 / 10$ pelo Comitê de Ética em Pesquisa da Secretaria de Estado de Saúde do Distrito Federal obtendo aprovação com o Parecer nº 283/2010.

\section{Análise Estatística}

As análises descritivas e a inferência foram realizadas através do programa estatístico SPSS 16 (IBM) com o apoio do Microsoft Office Excel 2007.

\section{RESULTADOS E DISCUSSÃO}

O grupo de estudo consistiu em 19 pacientes, sendo 9 $(47,3 \%)$ do sexo feminino e $10(52,6 \%)$ do sexo masculino, com a idade variando entre 36 e 74 anos $(20-39=21 \%$; $40-60=57,89 \%$; $>60=21 \%$ ). Quanto ao estado civil, 13
$(68,4 \%)$ se declararam casados ou que possuem uma união estável, 4 (21\%) são divorciados e $2(10,5 \%)$ solteiros. Em relação ao grau de escolaridade, $2(10,5 \%)$ são analfabetos, $8(42,1 \%)$ relataram ter cursado o ensino fundamental de forma incompleta, $3(10,5 \%)$ ensino fundamental completo, $2(10,5 \%)$ ensino médio completo, $1(5,2 \%)$ ensino técnico, $2(10,5 \%)$ ensino superior e $1(5,2 \%)$ não relatou sua situação educacional. Em relação à renda familiar, 14 (73\%) declararam 1 a 3 salários mínimos, 1 (5,2\%) declarou receber 3 a 5 salários mínimos, $2(10,5 \%)$ de 5 a 7 salários mínimos e $2(10,5 \%)$ mais de 7 salários mínimos por mês. A média de filhos foi menos de dois filhos por família. O tempo médio de acidente vascular encefálico foi 15,5 meses, variando de 6 a 31 meses. A média de tempo dos pacientes na terapia ocupacional é de 8,4 meses, variando de 2 a 18 meses de intervenção. Em pacientes com dominância do lado direito $(89,4 \%), 80 \%$ apresentaram hemiparesia no mesmo lado e dos pacientes com dominância esquerda $(10,5 \%)$, todos apresentam hemiparesia no mesmo lado (Tabela 1).

Tabela 1 - Características sócio demográficas dos pacientes

\begin{tabular}{|c|c|}
\hline Características sócio demográficas & Total $N=19$ \\
\hline Média de idade (DP) & $51,9(11,73)$ \\
\hline Faixa etária em anos & $36-74$ \\
\hline \multicolumn{2}{|l|}{ Sexo } \\
\hline Masculino & 10 \\
\hline Feminino & 9 \\
\hline \multicolumn{2}{|l|}{ Escolaridade } \\
\hline Não relata & 1 \\
\hline Analfabeto & 2 \\
\hline Fund.incompleto & 8 \\
\hline Fund.completo & 3 \\
\hline Médio completo & 2 \\
\hline Superior & 2 \\
\hline Técnico & 1 \\
\hline \multicolumn{2}{|l|}{ Renda familiar } \\
\hline De 1 a 3 SM & 14 \\
\hline De 3 a 5 SM & 1 \\
\hline De 5 a 7 SM & 2 \\
\hline Acima de $7 \mathrm{SM}$ & 2 \\
\hline \multicolumn{2}{|l|}{ Estado civil } \\
\hline Solteiro & 2 \\
\hline Casado & 10 \\
\hline Divorciado & 4 \\
\hline União estável & 3 \\
\hline \multicolumn{2}{|l|}{ AVE } \\
\hline Direito & 6 \\
\hline Esquerdo & 13 \\
\hline
\end{tabular}

Nota $:$ DP = desvio padrão; $\mathrm{AVE}=$ acidente vascular encefálico 
MENESES, K. V. P. et al. Perfil sócio demográfico e áreas. Rev. Ter. Ocup. Univ. São Paulo, v. 23, n. 2, p. 107-112, maio/ago. 2012.

Foram relatadas 40 áreas problema de desempenho ocupacional, divididas em autocuidado, produtividade e lazer. No item autocuidado, as áreas mais citados foram "alimentação" e "marcha" (citadas por 6 pacientes); no item produtividade, "cozinhar" e "pegar pequenas coisas/ movimento fino" (citadas por 5 pacientes) e no item lazer, “escrever" (citadas por 5 pacientes). Tais áreas estão apresentadas na Tabela 2 .

Tabela 2 - Problemas de desempenho apontados pelos sujeitos

\begin{tabular}{|c|c|c|}
\hline ÁREAS PROBLEMA & \multicolumn{2}{|c|}{ TOTAL } \\
\hline $\begin{array}{l}\text { 1. Autocuidado (cuidados pessoais, mobilidade funcional e independência } \\
\text { fora de casa). }\end{array}$ & $\begin{array}{l}N^{0} \text { vezes que a } \\
\text { atividade foi citada }\end{array}$ & Porcentagem (\%) \\
\hline Alimentação & 6 & 12,76 \\
\hline Andar/marcha & 6 & 12,76 \\
\hline Abotoar o botão do vestuário & 5 & 10,63 \\
\hline Higiene íntima & 5 & 10,63 \\
\hline Dirigir & 4 & 8,51 \\
\hline Pentear/amarrar o cabelo & 4 & 8,51 \\
\hline Vestir roupa & 4 & 8,51 \\
\hline Andar sozinha & 2 & 4,25 \\
\hline Fechar o sutiã & 2 & 4,25 \\
\hline Tomar banho & 2 & 4,25 \\
\hline Fazer uso da toalha & 2 & 4,25 \\
\hline Calçar meias & 1 & 2,1 \\
\hline Calçar sapatos & 1 & 2,1 \\
\hline Passar batom & 1 & 2,1 \\
\hline Prender cabelo & 1 & 2,1 \\
\hline Fazer uso do dinheiro & 1 & 2,1 \\
\hline Total & 47 & $100 \%$ \\
\hline \multicolumn{3}{|l|}{ 2. Produtividade (trabalho, tarefas domésticas e escola). } \\
\hline Cozinhar & 7 & 21,87 \\
\hline Pegar coisas pequenas/movimento fino & 5 & 15,62 \\
\hline Lavar roupa & 4 & 12,5 \\
\hline Pegar objetos maiores & 4 & 12,5 \\
\hline Varrer a casa & 3 & 9,37 \\
\hline Costurar & 2 & 6,25 \\
\hline Cortar cabelo & 1 & 3,12 \\
\hline Cortar verduras & 1 & 3,12 \\
\hline Descascar & 1 & 3,12 \\
\hline Dobrar roupas & 1 & 3,12 \\
\hline Escrever com a mão direita & 1 & 3,12 \\
\hline Pegar objetos no armário & 1 & 3,12 \\
\hline Voltar a trabalhar (carregar botija de gás) & 1 & 3,12 \\
\hline Total & & $100 \%$ \\
\hline \multicolumn{3}{|l|}{ 3. Lazer (recreação ativa, tranquila e socialização). } \\
\hline Escrever & 5 & 29,41 \\
\hline Dificuldade na fala & 2 & 23,52 \\
\hline Lentidão de leitura & 2 & 23,52 \\
\hline Cuidar das plantas & 1 & 5,88 \\
\hline Dançar & 1 & 5,88 \\
\hline Fazer crochê & 1 & 5,88 \\
\hline Jogar bola & 1 & 5,88 \\
\hline Pescar & 1 & 5,88 \\
\hline Segurar revista/jornal & 1 & 5,88 \\
\hline Tocar violão & 1 & 5,88 \\
\hline Usar o computador & 1 & 5,88 \\
\hline Total & & $100 \%$ \\
\hline
\end{tabular}

A relação entre as áreas de desempenho mais afetadas e o perfil sócio demográfico da população do estudo nos levou a apontamentos sobre diversos fatores que podem dificultar o processo de reabilitação desses 
pacientes. De acordo com Lavados et al. (2007) o baixo nível socioeconômico está relacionado ao pouco acesso ao conhecimento e aos serviços de saúde, a um estilo de vida não saudável, à baixa adesão às estratégias de prevenção, inadequadas condições de moradia e aumento do estress. Estes fatores também interferem no processo de reabilitação destes pacientes. Levando em consideração o perfil da população deste estudo, que são indivíduos com baixa escolaridade, de baixa renda, sendo em maior parte composta por homens em idade produtiva, responsável pela subsistência de sua família e que apresentam variadas limitações devido ao AVE, pode-se perceber o impacto das alterações provocadas pela patologia em seu estilo de vida e de sua família. Foi observado a perda do papel de provedor da família, a incapacidade em realizar atividades que antes eram comuns e rotineiras como se alimentar, se vestir, dirigir, escrever, fazer uso adequado do dinheiro, trabalhar, etc., o sentimento de inutilidade, de baixa estima, de sobrecarga familiar e desmotivação para viver. Estas alterações afetam diretamente a autonomia, a independência e a participação social dos pacientes. A família também foi afetada, muitas vezes ocorrendo inversões de papéis, alteração da rotina e da renda familiar e modificação na participação social.

Dificuldades relatadas pelos pacientes como a falta de suporte social ou o suporte inadequado, como tempo longo de espera para início do tratamento de reabilitação, número de sessões limitadas pelo serviço (falta de profissionais, número reduzido de vagas, espaço físico inadequado, falta de recursos financeiros e materiais), dificuldade em acesso aos equipamentos que auxiliam no processo de reabilitação e retorno da independência (cadeira de rodas, andadores, órteses e outras adaptações para possibilitar o desempenho nas tarefas do dia a dia), falta de acessibilidade (transporte público inadequado, calçadas e vias apresentando barreiras arquitetônicas) parece interferir diretamente no potencial de recuperação do paciente e no retorno ao desempenho ocupacional.

Diante deste contexto, percebe-se a importância do suporte familiar e social, bem como da estrutura adequada dos serviços de reabilitação para que o sujeito possa atingir, por meio do tratamento reabilitador ou compensatório, seu potencial de reabilitação.

\section{CONSIDERAÇÕES FINAIS}

Este estudo mostrou o perfil sócio demográfico de indivíduos pós AVE atendidos por um serviço de terapia ocupacional e identificou as áreas de desempenho ocupacional mais afetadas. Estes resultados apontam para a importância de abordagens reabilitadoras que foquem nestas áreas de desempenho mais afetadas pelo AVE, de forma a possibilitar maior independência, autonomia e participação social, além de mostrar a importância do suporte social e familiar na recuperação do indivíduo.

O conhecimento do perfil sócio demográfico desta clientela é fundamental para o planejamento de estratégias adequadas de reabilitação, bem como orientar a tomada de decisões relativas ao planejamento dos serviços de saúde e sociais e a distribuição de recursos humanos e financeiros, assegurando a saúde como direito e dever do Estado.

MENESES, K. V. P.; DUARTE, J. S.; ALENCAR, V. O.; PEREIRA, A. C. S. Socio-demographic profile and areas of occupational performance in stroke patients attended by occupational therapy service. Rev. Ter. Ocup. Univ. São Paulo, v. 23, n. 2, p. 107-112, maio/ago. 2012.

\begin{abstract}
Background: Stroke (cerebrovascular accident; CVA) resulting in difficulty or inability to perform functional tasks and daily tasks, making it difficult or impossible to return to work, participation in family and community. Occupational therapy interventions help to improve skills in activities of daily life, encouraging greater independence, autonomy, social participation and quality of life. Objective: To identify socio-demographic profile of patients after stroke treated in an occupational therapy service and identify the filds most affected occupational performance. Methodology: Cross-sectional study with patients with stroke. We used an interview to collect sociodemographic information and the Canadian Occupational Performance Measure (COPM). Results: 19 patients, $9(47.3 \%)$ were female and $10(52.6 \%)$ were male, aged between 36 and 74 years $(20-39=21 \%, 40-60=57.89 \%,>60=21 \%) .13(68.4 \%)$ were married or had stable union. Most had finished elementary school. Individuals reported 40 areas of occupational performance problems, the most quoted food and gear (self-care), cooking (productivity) and write (leisure activities). Conclusion: The results indicate the importance of rehabilitative approaches that focus on performance in these areas most affected by stroke, in order to enable greater independence, autonomy and social participation.
\end{abstract}

KEY WORDS: Stroke/rehabilitation; Stroke/therapy; Sickness impact profile; Occupational therapy. 


\section{REFERÊNCIAS}

ANDRÉ, C.; CURIONI, C. C.; BRAGADA CUNHA, C.; VERAS, R. Progressive decline in stroke mortality in Brazil from 1980 to 1982,1990 to 1992 , and 2000 to 2002. Stroke, v. 37, p. 27842789, 2006.

ARES, M. J. J. Acidente vascular encefálico. In: TEIXEIRA, E. Terapia ocupacional na reabilitação física. São Paulo: ROCCA, 2003. Cap.1, p. 3-15.

BARROS, J. E. F. Doença encefalovascular. In: BARROS, J. E. F.; NITRINI, R.; BACHESCHI, L. A. A neurologia que todo médico deve saber. 2. ed. São Paulo: Atheneu, 2003. p.171-188.

CRUZ, D. M. C.; TOYODA, C. Y. Terapia ocupacional no tratamento do AVC. UNICAMP: ComCiência, v. 109, p. 1-5, 2009.

CRUZ, K. C. T.; DIOGO, M. J. D. Avaliação da capacidade funcional de idosos com acidente vascular encefálico. Acta Paul. Enferm., v. 22, n. 5, p. 666-672, 2009.

CURIONI, C.; CUNHA, C. B.; VERAS, R. P.; ANDRÉ, C. The decline in mortality from circulatory diseases in Brazil. Rev. Panam. Salud Publica; v. 25, n. 1, p.9-15, 2009.

DELBONI, M. C. C., et al. Relação entre os aspectos das alterações funcionais e seu impacto na qualidade de vida das pessoas com sequelas de acidente vascular encefálico (AVE). Mundo da Saúde, São Paulo, v. 34, n. 2, p. 165-175, 2010.

DOBKIN, B. Strategies for stroke rehabilitation. Lancet Neurol., v. 3, p. 528-536, 2004;

LAW, M., et al. Medida Canadense de Desempenho Ocupacional (COPM). Trad. A. A. CARDOSO, L. V. MAGALHÃES, L. C. MAGALHÃES. Belo Horizonte: Ed UFMG, 2009.

LAVADOS, P. M.; et al. Stroke epidemiology, prevention, and management strategies at a regional level: Latin America and the Caribbean. Lancet Neurol., v. 6, p. 362-372, 2007.

PEREIRA, A. B. C. N. G.; et al. Prevalência de acidente vascular cerebral em idosos no Município de Vassouras, Rio de Janeiro, Brasil, através do rastreamento de dados do Programa Saúde da Família. Cad. Saúde Pública, v. 25, n. 9, p. 1929-1936, 2009.

POlESE, J. C; TONIAL, A; JUNG, F. K; MAZUCO, R.; OLIVEIRA, S. G.; SCHUSTER, R. C. Avaliação da funcionalidade de indivíduos acometidos por acidente vascular encefálico. Rev. Neurocienc. v. 16, n. 3, p. 175-178, 2008.

Recebido para publicação: 27/01/12

Aceito para publicação: 29/06/2012 